\title{
Lung ultrasound score to determine the effect of fraction inspired oxygen during alveolar recruitment on absorption atelectasis in laparoscopic surgery: a randomized controlled trial
}

\author{
Bo Rim Kim, Seohee Lee, Hansu Bae, Minkyoo Lee, Jae-Hyon Bahk and Susie Yoon * (])
}

\begin{abstract}
Background: Although the intraoperative alveolar recruitment maneuver (RM) efficiently treats atelectasis, the effect of $\mathrm{FIO}_{2}$ on atelectasis during $\mathrm{RM}$ is uncertain. We hypothesized that a high $\mathrm{FlO}_{2}(1.0)$ during $\mathrm{RM}$ would lead to a higher degree of postoperative atelectasis without benefiting oxygenation when compared to low $\mathrm{FlO}_{2}(0.4)$.

Methods: In this randomized controlled trial, patients undergoing elective laparoscopic surgery in the Trendelenburg position were allocated to low- $\left(\mathrm{FIO}_{2} 0.4, n=44\right)$ and high- $\mathrm{FIO}_{2}\left(\mathrm{FlO}_{2} 1.0, n=46\right)$ groups. RM was performed 1-min post tracheal intubation and post changes in supine and Trendelenburg positions during surgery. We set the intraoperative $\mathrm{FlO}_{2}$ at 0.4 for both groups and calculated the modified lung ultrasound score (LUSS) to assess lung aeration after anesthesia induction and at surgery completion. The primary outcome was modified LUSS at the end of the surgery. The secondary outcomes were the intra- and postoperative $\mathrm{PaO}_{2}$ to $\mathrm{FlO}_{2}$ ratio and postoperative pulmonary complications.

Results: The modified LUSS before capnoperitoneum and RM $(P=0.747)$ were similar in both groups. However, the postoperative modified LUSS was significantly lower in the low $\mathrm{FlO}_{2}$ group (median difference 5.0, 95\% Cl 3.0-7.0, $P<0.001$ ). Postoperatively, substantial atelectasis was more common in the high- $\mathrm{FIO}_{2}$ group (relative risk $1.77,95 \%$ $\mathrm{Cl} 1.27-2.47, \mathrm{P}<0.001$ ). Intra- and postoperative $\mathrm{PaO}_{2}$ to $\mathrm{FlO}_{2}$ were similar with no postoperative pulmonary complications. Atelectasis occurred more frequently when RM was performed with high than with $\mathrm{low}^{\mathrm{FiO}}{ }_{2}$; oxygenation was not benefitted by a high- $\mathrm{FlO}_{2}$.

Conclusions: In patients undergoing laparoscopic surgery in the Trendelenburg position, absorption atelectasis occurred more frequently with high rather than low $\mathrm{FiO}_{2}$. No oxygenation benefit was observed in the high- $\mathrm{FlO}_{2}$ group.
\end{abstract}

Trial registration: ClinicalTrials.gov, NCT03943433. Registered 7 May 2019,

Keywords: Alveolar recruitment, Lung, Oxygen, Pulmonary atelectasis, Ultrasonography

\footnotetext{
*Correspondence: susiey87@gmail.com

Department of Anesthesiology and Pain Medicine, Seoul National University Hospital, Seoul National University College of Medicine, 101 Daehak-ro,

Jongno-gu, Seoul 03080, Republic of Korea
}

(c) The Author(s). 2020 Open Access This article is licensed under a Creative Commons Attribution 4.0 International License, which permits use, sharing, adaptation, distribution and reproduction in any medium or format, as long as you give appropriate credit to the original author(s) and the source, provide a link to the Creative Commons licence, and indicate if changes were made. The images or other third party material in this article are included in the article's Creative Commons licence, unless indicated otherwise in a credit line to the material. If material is not included in the article's Creative Commons licence and your intended use is not permitted by statutory regulation or exceeds the permitted use, you will need to obtain permission directly from the copyright holder. To view a copy of this licence, visit http://creativecommons.org/licenses/by/4.0/. The Creative Commons Public Domain Dedication waiver (http://creativecommons.org/publicdomain/zero/1.0/) applies to the data made available in this article, unless otherwise stated in a credit line to the data. 


\section{Background}

During general anesthesia, atelectasis reportedly occurs in most patients [1], typically due to absorption of gas, compression of the lung tissue, and impairment of surfactant function [2]. Additionally, during laparoscopic surgery, the increased abdominal pressure of capnoperitoneum may shift the diaphragm cranially and decrease respiratory compliance $[3,4]$. Compression of basal lung regions due to a stiffened diaphragm would accelerate the formation of atelectasis that was already initiated during anesthesia induction [4]. Moreover, the steep Trendelenburg position used in laparoscopic gynecologic or colon surgery causes the abdominal contents to push the diaphragm more cephalad, resulting in aggravated lung collapse and decreased functional residual capacity $[5,6]$.

Intraoperative atelectasis is associated with decreased lung compliance, impaired oxygenation, increased pulmonary vascular resistance, and lung injury $[5,7]$. Moreover, atelectasis can persist postoperatively and result in respiratory complications, such as hypoxemia and infection, significantly impacting patient recovery $[5,8]$.

The alveolar recruitment maneuver (RM) with positive-end expiratory pressure (PEEP) has been advocated as efficient for atelectasis treatment [9-13]. Reports on the impact of $\mathrm{FIO}_{2}$ during RM on atelectasis development are rare, and have not limited $\mathrm{FIO}_{2}$ to the RM per se [14]. While RM with high $\mathrm{FIO}_{2}$ can improve oxygenation rapidly, there is a greater possibility of absorption atelectasis occurring.

Although computed tomography has been considered as the gold standard for lung imaging, it is less optimal for routine examination of perioperative atelectasis due to the cumbersomeness and the risk of radiation exposure. On the other hand, lung ultrasound is a portable, non-invasive, and radiation-free device $[15,16]$. Recent studies have shown the utility of the lung ultrasound score (LUSS) in the operating room [15-19]. The diagnostic reliability of LUSS for detecting perioperative atelectasis has been verified against computed tomography or magnetic resonance imaging $[15,19]$.

We prospectively assessed the impact of $\mathrm{FIO}_{2}$, specifically during RM, on atelectasis development, using the LUSS. We hypothesized that during RM, a high $\mathrm{FIO}_{2}$ (1.0) leads to a higher risk for postoperative atelectasis in adults undergoing laparoscopic surgery, without benefiting oxygenation, than low $\mathrm{FIO}_{2}(0.4)$.

\section{Methods}

\section{Design}

This prospective, patient- and sonographer-blinded, single-center, parallel, randomized, controlled trial was approved by the Institutional Review Board of Seoul National University Hospital (No. 1903-137-1020, 22 April 2019) and registered at ClinicalTrials.gov (NCT03943433, 7 May 2019). The study was conducted in accordance with CONSORT guidelines. We enrolled adult patients scheduled to undergo elective laparoscopic gynecologic surgery or colorectal surgery in the Trendelenburg position from May to November 2019 after obtaining written informed consent. The inclusion criterion was adult patients aged 20-70 years with an American Society of Anesthesiologists physical status III. The exclusion criteria were patients with body mass index $\geq 35 \mathrm{~kg} \mathrm{~m}^{-2}$, cardiovascular impairment, severe chronic obstructive pulmonary disease (preoperative forced expiratory volume in $1 \mathrm{~s}$ /forced vital capacity of $60 \%$ or lower) or emphysema, pneumothorax or bullae, previous lung resection surgery, and increased intracranial pressure. Some patients dropped out because of protocol violation, massive bleeding with hemodynamic compromise, or unexpected open conversion.

Patients were randomly assigned to two groups based on the applied $\mathrm{FIO}_{2}$ during $\mathrm{RM}$, in a $1: 1$ ratio, by computer-generated randomization, using $\mathrm{R}$ software (version 3.5.1, R Foundation for Statistical Computing, Vienna, Austria). Allocation was concealed in an opaque envelope by an assistant not involved in the study and was delivered to the attending anesthesiologist before general anesthesia induction. The sonographer (BRK or HB) was completely blinded to the group assignment.

\section{Anesthesia and ventilator strategy}

General anesthesia was induced according to the predetermined protocol with standard monitoring of pulse oximetry $\left(\mathrm{SPO}_{2}\right)$, non-invasive blood pressure, electrocardiography, bispectral index (A-2000 XP; Aspect Medical Systems, Newton, MA), and end-tidal carbon dioxide concentration. After preoxygenation with 100\% oxygen, propofol $1.5-2.0 \mathrm{mg} \mathrm{kg}^{-1}$ was administered intravenously with a continuous target-controlled remifentanil infusion (Orchestra; Fresenius Kabi, Brézins, France). Rocuronium $0.6-0.8 \mathrm{mg} \mathrm{kg}^{-1}$ was administered for neuromuscular blockade, and tracheal intubation was performed. General anesthesia was maintained with sevoflurane and remifentanil to maintain the bispectral index within 40-60. A radial arterial catheter was placed and connected to an arterial waveform analysis system (Flotrac; Edwards Lifesciences, Irvine, CA) for close monitoring of intraoperative hemodynamic changes derived from the RM, as a part of the institutional protocols.

Mechanical ventilation was maintained intraoperatively with the $\mathrm{FIO}_{2}$ at 0.4 , tidal volume at $8 \mathrm{ml} \mathrm{kg}^{-1}$ of ideal body weight, PEEP at $5 \mathrm{cmH}_{2} \mathrm{O}$, inspiration to 
expiration ratio of 1:2, and end-inspiration pause $10 \%$ at volume-controlled ventilation mode. Respiratory rate was adjusted to maintain partial pressure of arterial carbon dioxide at $35-45 \mathrm{mmHg}$. If the peak airway pressure exceeded $35 \mathrm{cmH}_{2} \mathrm{O}$, the tidal volume was decreased stepwise by $1 \mathrm{ml} \mathrm{kg}^{-1}$ until the peak pressure was $<35 \mathrm{cmH}_{2} \mathrm{O}$.

At the end of the surgery, sugammadex $2-4 \mathrm{mg} \mathrm{kg}^{-1}$ was administered after train-of-four count monitoring for reversal of neuromuscular blockade. The $\mathrm{FiO}_{2}$ was changed to 1.0 when the first spontaneous breathing was observed. After extubation, patients were transferred to the post-anesthesia care unit (PACU). Intravenous patient-controlled analgesia was routinely used for postoperative pain control. Patients were discharged from the PACU when they met the Modified Aldrete Score criteria [20].

\section{Lung ultrasound examination and RM strategy}

Lung ultrasound examination was performed at three time-points: $1 \mathrm{~min}$ after starting mechanical ventilation, at the end of surgery (before emergence), and after breathing room air for $20 \mathrm{~min}$ at PACU (Fig. 1). Lung ultrasound was performed by two investigators (BRK and $\mathrm{HB}$ ) blinded to the group assignment. Both investigators had performed more than 100 cases of lung ultrasound. The ultrasound was performed in the supine position using a Vivid-I ultrasound device (GE Healthcare, Chalfont St. Giles, Bucks, UK) and a convex probe, with a frequency of $2.5 \mathrm{MHz}-7.5 \mathrm{MHz}$. All intercostal spaces were examined as previously described: each hemithorax was divided into six regions with three longitudinal lines (parasternal, anterior, and posterior axillary) and two axial lines (one above the diaphragm and another at $1 \mathrm{~cm}$ above the nipples) [15]. Each region was scored according to the modified LUSS system suggested by Monastesse et al., which showed sufficient sensitivity to detect loss of aeration during laparoscopic surgery [21]. The degree of deaeration was rated from 0 to 3 as follows (Fig. 2): $0,0-2$ B lines; $1, \geq 3 \mathrm{~B}$ lines or 1 or multiple subpleural consolidations separated by a normal pleural line; 2 , multiple coalescent B lines or multiple subpleural consolidations separated by a thickened or irregular pleural line; and 3, consolidation or small subpleural consolidation exceeding $1 \times 2 \mathrm{~cm}$ in diameter [21] The points for the 12 regions were summed for analysis. Furthermore, we defined substantial atelectasis as a score of 2 or 3 assigned to any region.

RM was performed after lung ultrasound examinations (twice) under real-time ultrasound guidance, with the probe placed at the region with the highest score. After setting the $\mathrm{FIO}_{2}$ (1.0 or 0.4) according to the assignment, continuous positive airway pressure was applied from 15
$\mathrm{cmH}_{2} \mathrm{O}$ in $5-\mathrm{cmH}_{2} \mathrm{O}$ stepwise increments, up to the pressure at which no collapsed area was observed. The maximum continuous airway pressure applied during RM was $40 \mathrm{cmH}_{2} \mathrm{O}$. The applied pressure (opening pressure) and the duration of the RM were recorded. Additional intraoperative RM was performed at several time-points: at the time of Trendelenburg positioning and at every $30 \mathrm{~min}$ thereafter, and after a return to supine position after procedure completion (Fig. 1). Intraoperative RM was performed using the initial pressure and duration after adjustment of $\mathrm{FiO}_{2}$ according to the group assignment. The pre-designated $\mathrm{FIO}_{2}$ was applied only during the RM, after which it was maintained at 0.4 throughout mechanical ventilation in both groups.

\section{Outcomes}

The primary outcome was the modified LUSS at surgery completion (before emergence), reflecting an aeration loss during general anesthesia. The secondary outcomes were the modified LUSS at PACU, substantial atelectasis observed on lung ultrasound, intraoperative and postoperative $\mathrm{PaO}_{2}$ to $\mathrm{FIO}_{2}$ ratios, and incidences of intraoperative desaturation $\left(\mathrm{SPO}_{2}<95 \%\right)$, postoperative fever (body temperature $>38^{\circ} \mathrm{C}$ during hospital stay), and postoperative pulmonary complications during hospital stay. Arterial blood samples were obtained $20 \mathrm{~min}$ after a change in position from supine to Trendelenburg and after breathing room air for $20 \mathrm{~min}$ at the PACU. Postoperative atelectasis, pneumonia, acute respiratory distress syndrome, and pulmonary aspiration data were collected by reviewing medical records. Their severity was evaluated based on previous consensus definitions for standardized perioperative pulmonary complications [22]. In our study, in-hospital pulmonary complications were atelectasis, pneumonia, acute respiratory distress syndrome and mild-to-severe pulmonary aspiration. Data on postoperative pulmonary complications were collected during the hospital stay. Additionally, data on age, height, weight, sex, type of operation, duration of anesthesia and surgery, pressure and duration of RM, and ventilator parameters were collected. Significant hemodynamic deterioration during RM (> 20\% of baseline) was documented and treated with vasoactive drugs or crystalloid agents.

\section{Statistical analysis}

In our pilot study on patients undergoing laparoscopic surgery in the Trendelenburg position $(n=10)$, the modified LUSS [mean (SD)] before and at the end of surgery were 3.88 (1.26) and 8.66 (2.82), respectively. Considering a $20 \%$ decrease in the modified LUSS in the low $\mathrm{FIO}_{2}$ group, we calculated that 44 patients would be 


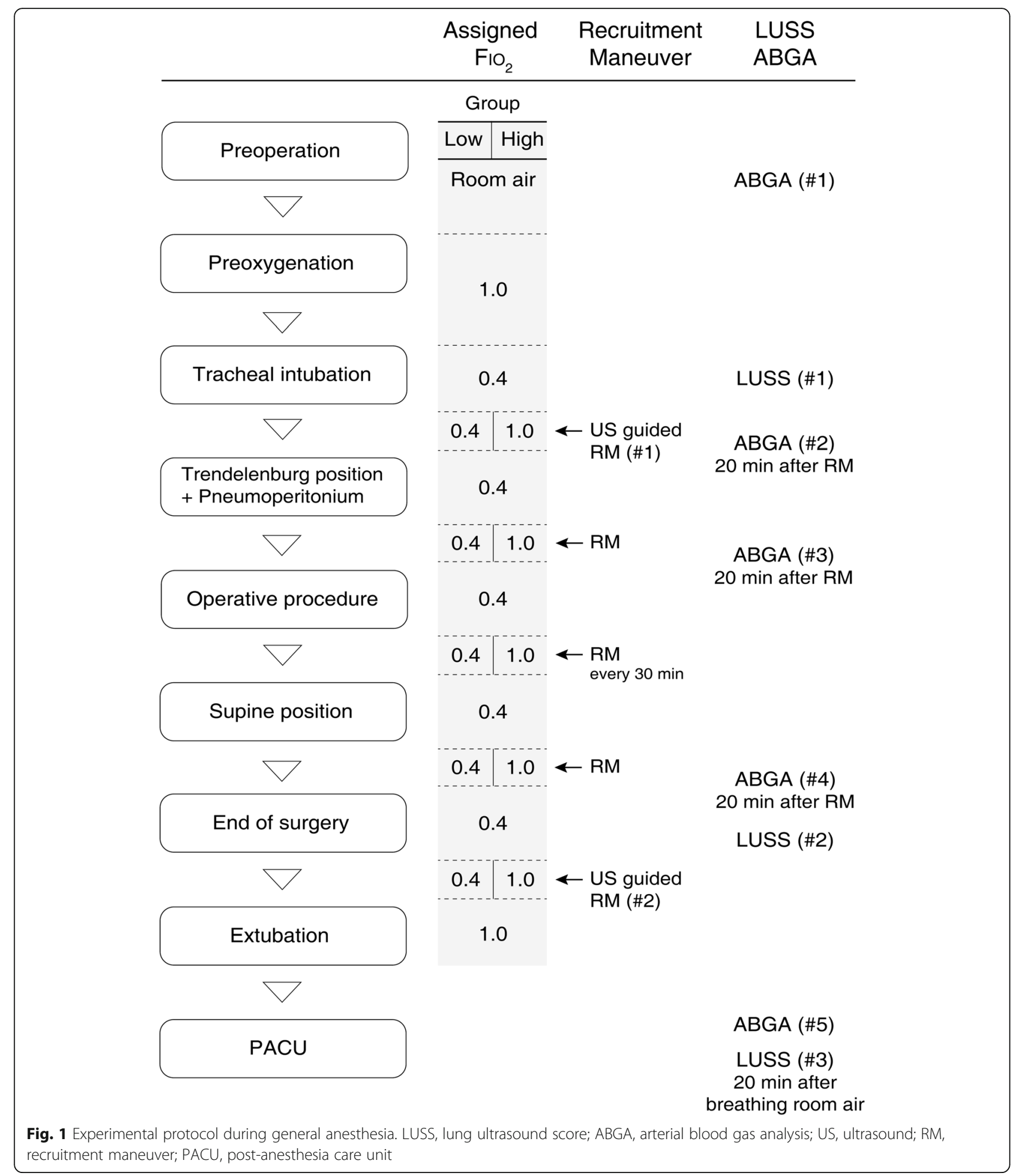

needed in each group, with a type-I error risk of 0.05 and a power of 0.8 for two-tailed analysis.

Continuous variables were summarized as mean (SD) or median (interquartile range). The variables were analyzed using unpaired or paired $t$-tests and the Mann-Whitney $\mathrm{U}$ or Wilcoxon signed-rank tests, after assessing the normality of data distribution with the Shapiro-Wilk test. Number of patients (\%) was compared using the chisquared test or Fisher's exact test. Statistical analyses were performed with R software (version 3.5.1, R Foundation for Statistical Computing, Vienna, Austria). For all analyses, $P<0.05$ was statistically significant. 

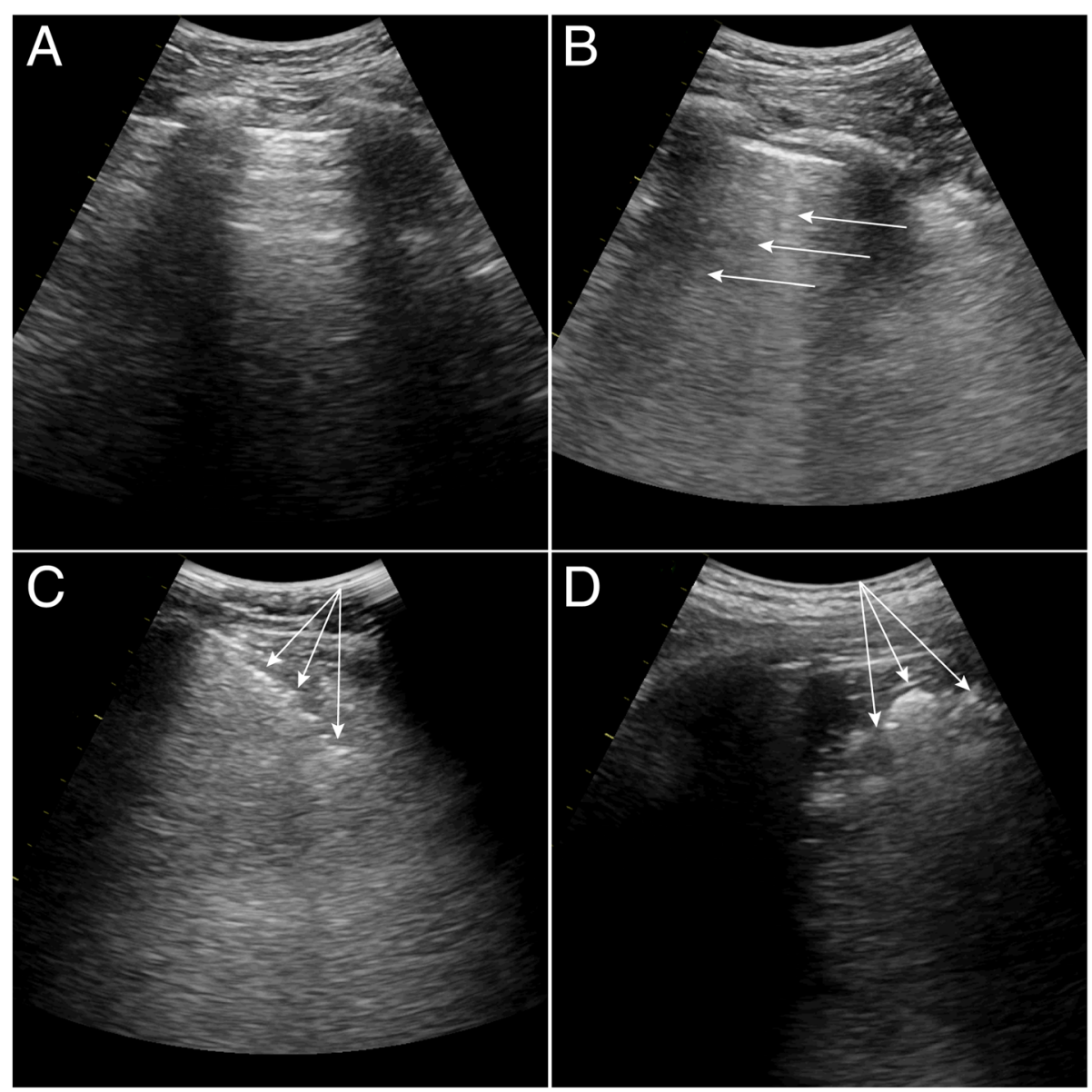

Fig. 2 Lung ultrasound findings with different scores. Modified lung ultrasound scoring system in accordance with the method of Monastesse et al. (A) Normal pattern 'bat-sign' with A-lines parallel to the pleural line, score =0; (B) More than three B lines arising from pleural line, score = 1; (C) Multiple subpleural consolidations separated by an irregular pleural line, score $=2$; (D) Large-sized consolidation, score $=3$. Each arrow indicates pathologic findings of each figure

\section{Results}

One-hundred-and-seventy-eight patients scheduled to undergo laparoscopic surgery in the Trendelenburg position were assessed for eligibility. Among them, 98 patients met the inclusion criteria and were randomized to the low- $(n=49)$ or the high- $\mathrm{FiO}_{2}$ groups $(\mathrm{n}=49)$. Five patients in the low- $\mathrm{FIO}_{2}$ and two patients in the high$\mathrm{FIO}_{2}$ group dropped out owing to an intraoperative change to supine position. One patient was excluded owing to an ultrasound machine breakdown. Consequently, 44 and 46 patients in each group were analyzed, respectively (Fig. 3).

Participants' baseline characteristics are summarized in Table 1. The groups did not differ in terms of patient characteristics or operational data. The modified LUSS are presented in Table 2. The baseline modified LUSS, measured at $1 \mathrm{~min}$ after anesthesia induction did not differ between the groups $(P=0.747)$. For the primary outcome, the modified LUSS at the end of surgery was significantly lower in the low- $\mathrm{FIO}_{2}$ group (median difference 5.0, 95\% CI 3.0-7.0, $P<0.001$ ). Moreover, the modified LUSS at $20 \mathrm{~min}$ after breathing room air at the PACU was significantly lower in the low- $\mathrm{FIO}_{2}$ group $(P<0.001)$. Substantial atelectasis at $1 \mathrm{~min}$ after starting mechanical ventilation was observed in $12(27.3 \%)$ and 15 (32.6\%) patients in the low- and high- $\mathrm{FIO}_{2}$ groups, respectively $(P=0.747)$. However, this was more frequently observed in the high- $-\mathrm{FIO}_{2}$ after surgery completion (relative risk 1.77, 95\% CI 1.27-2.47, $P<0.001$ ) and at PACU (relative risk 1.73, 95\%CI 1.26-2.38, $P<0.001$ ).

The perioperative $\mathrm{PaO}_{2}$ to $\mathrm{FIO}_{2}$ ratio did not differ between the groups at any time-point (Table 3). The incidence of intraoperative desaturation and the lowest $\mathrm{SPO}_{2}$ value during anesthesia did not differ between the groups $(P=0.959$ and $P=0.119$, respectively) (Table 4$)$. Hemodynamic and respiratory variables in the Trendelenburg position with capnoperitoneum are summarized in Table 4. 


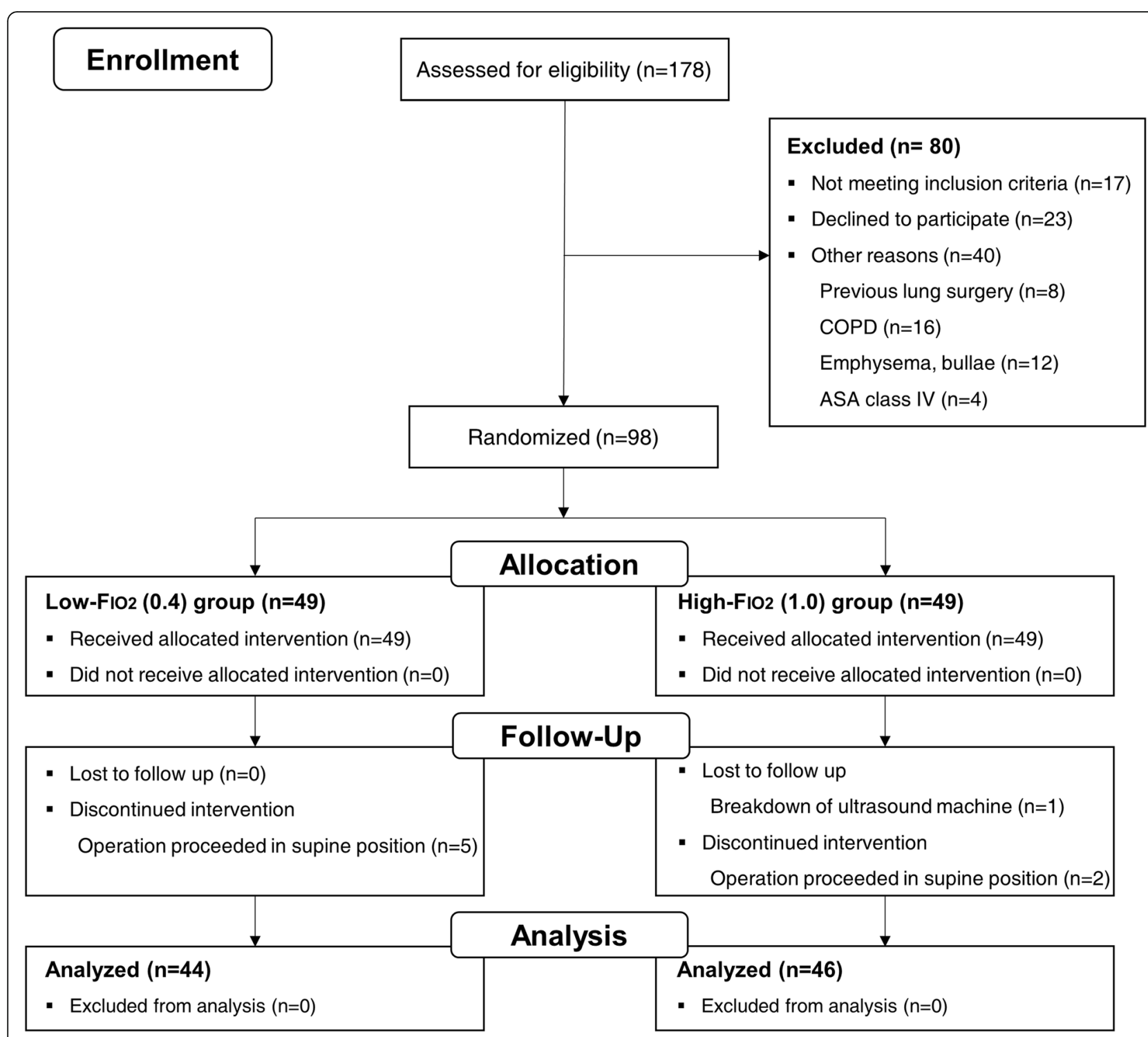

Fig. 3 CONSORT diagram. COPD, chronic obstructive pulmonary disease; ASA, American Society of Anesthesiologists

The opening pressure for the RM varied from 25 to 40 $\mathrm{cmH}_{2} \mathrm{O}$ and was similar between groups $(P=0.773)$. For 38 patients in the low- $\mathrm{FIO}_{2}$ group $(86.4 \%)$ and 40 patients in the high- $\mathrm{FIO}_{2}$ group (87.0), $30 \mathrm{cmH}_{2} \mathrm{O}$ was used to resolve the atelectasis. An opening pressure of 35 $\mathrm{cmH}_{2} \mathrm{O}$ was needed for four $(9.1 \%)$ and for five (10.9\%) patients in the low- $-\mathrm{FIO}_{2}$ and high- $\mathrm{FIO}_{2}$ groups, respectively. For one patient in each group, an opening pressure of $25 \mathrm{cmH}_{2} \mathrm{O}$ was required. One patient in the low- $\mathrm{FIO}_{2}$ group required $40 \mathrm{cmH}_{2} \mathrm{O}$ to restore all collapsed areas. Hemodynamic deterioration was observed in 21 (47.7\%) and 20 (43.5\%) patients during RM in the low- and high-FIO 2 groups, respectively $(P=0.687)$.
No postoperative pulmonary complication was reported during hospital stay. (Table 4). Five (9.1\%) and 3 (6.5\%) patients showed subsegmental atelectasis on postoperative radiographs in the low- and high- $\mathrm{FIO}_{2}$ groups, respectively $(P=0.710)$. Postoperative fever $\left(>38^{\circ} \mathrm{C}\right)$ occurred in $17.8 \%$ of the study population, with a similar incidence between the 2 groups $(P=0.317)$.

\section{Discussion}

This study evaluated the impact of $\mathrm{FIO}_{2}$ during $\mathrm{RM}$ on development of postoperative atelectasis, using lung ultrasound. The postoperative modified LUSS was higher in the high- $\mathrm{FIO}_{2}$ group, indicating more severe 
Table 1 Characteristics of patients, surgery, and anesthesia

\begin{tabular}{|c|c|c|c|}
\hline & Low-FIO 2 group $(n=44)$ & High-FıO 2 group $(n=46)$ & $P$-value \\
\hline Age (year) & $49.5(43.0-59.0)$ & $54.5(43.0-61.0)$ & 0.508 \\
\hline Female, $\mathrm{n}$ & $32(72.7)$ & $28(60.9)$ & 0.332 \\
\hline Height (cm) & $158.5(154.7-165.5)$ & $161.4(156.0-170.0)$ & 0.184 \\
\hline Weight (kg) & $61.7(54.0-68.9)$ & $60.5(54.0-70.0)$ & 0.987 \\
\hline Predicted body weight (kg) & $52.0(48.0-59.5)$ & $54.0(48.0-66.0)$ & 0.140 \\
\hline Body mass index $\left(\mathrm{kg} \mathrm{m}^{-2}\right)$ & $23.7(21.8-26.2)$ & $24.1(21.0-25.9)$ & 0.617 \\
\hline ASA classification (I/II), n & $31 / 13$ & $24 / 22$ & 0.118 \\
\hline \multicolumn{4}{|l|}{ Comorbidity } \\
\hline Hypertension, n & $9(20.5)$ & $11(23.9)$ & 0.888 \\
\hline Diabetes mellitus, n & $3(6.8)$ & $7(15.2)$ & 0.351 \\
\hline Current Smoker, n & $4(9.1)$ & $7(15.2)$ & 0.572 \\
\hline ARISCAT score & $23(8-26)$ & $19(7-26)$ & 0.322 \\
\hline Type of surgery & & & 0.406 \\
\hline Laparoscopic colorectal surgery, n & $21(47.7)$ & $27(58.7)$ & \\
\hline Laparoscopic gynecologic surgery, n & $23(52.3)$ & $19(41.3)$ & \\
\hline \multicolumn{4}{|l|}{ Operative profiles } \\
\hline Duration of anesthesia (min) & $147.5(107.5-195.5)$ & $170.0(115.0-230.0)$ & 0.109 \\
\hline Duration of surgery (min) & $100.0(70.0-140.0)$ & $125.0(85.0-180.0)$ & 0.058 \\
\hline Duration of Trendelenburg position (min) & $70.0(46.5-100.5)$ & $80.0(56.0-142.0)$ & 0.054 \\
\hline Intraoperative crystalloid administration (ml) & $600.0(500.0-875.0)$ & $700.0(400.0-1000.0)$ & 0.484 \\
\hline Estimated blood loss (ml) & $65.0(40.0-112.5)$ & $100.0(50.0-200.0)$ & 0.145 \\
\hline Urine output (ml) & $130.0(80.0-200.0)(n=39)^{*}$ & $150.0(85.0-265.0)(n=43)^{*}$ & 0.111 \\
\hline Intraoperative inotropic requirement, n & $20.0(45.4)$ & $28.0(60.9)$ & 0.356 \\
\hline
\end{tabular}

Values are expressed as median (Interquartile range) or number (\%). ASA, American Society of Anesthesiologists; ARISCAT, Assess Respiratory Risk in Surgical patients in Catalonia. *Urine output was measured in patients with Foley catheter

aeration loss in this group. In addition, postoperative consolidation was more frequently observed in the high$\mathrm{FIO}_{2}$ group, with no significant difference in the preoperative modified LUSS. Oxygenation was similar between groups at any time-point. These observations were consistent with our hypothesis that using a high $\mathrm{FIO}_{2}$ (1.0) during RM would not benefit oxygenation and lead to more postoperative atelectasis than using a low $\mathrm{FIO}_{2}(0.4)$.

High $\mathrm{FIO}_{2}$ is associated with the development of absorption atelectasis during general anesthesia [23, 24]. However, to the best of our knowledge, the impact of a temporary high $\mathrm{FIO}_{2}$ during RM on atelectasis development has not been investigated. In this study, patients assigned to the high- $\mathrm{FIO}_{2}$ group received RM with $\mathrm{FIO}_{2}$ 1.0, whereas those in the low- $\mathrm{FIO}_{2}$ group received RM with $\mathrm{FIO}_{2}$ 0.4. The $\mathrm{FIO}_{2}$ was uniformly maintained at 0.4 with 5- $\mathrm{cmH}_{2} \mathrm{O}$ PEEP during post- $\mathrm{RM}$ mechanical ventilation in both groups. A high oxygen concentration in the alveoli during RM was predicted to cause increased absorption atelectasis. Consequently, the postoperative modified LUSS was significantly lower in the low-FIO with the difference persisting in the PACU.

Using computed tomography, Rothen et al. demonstrated the progression of absorption atelectasis over time after RM in 12 patients, with an $\mathrm{FIO}_{2}$ of 0.4 or 1.0 during RM and thereafter [25]. Absorption atelectasis developed within 5 min in the $\mathrm{FiO}_{2} 1.0$ group and after $40 \mathrm{~min}$ in the $\mathrm{FiO}_{2}$ 0.4 group. Although the impact of oxygen concentration was obvious, this and the present study differed in that the previous study applied the designated $\mathrm{FIO}_{2}$ not only during $\mathrm{RM}$, but also during the rest of the study period. Additionally, Song et al. studied absorption atelectasis based on the $\mathrm{FIO}_{2}$ during mechanical ventilation, using lung ultrasound in children [14]. Although the $\mathrm{FIO}_{2}$ had no significant impact on the incidence of significant atelectasis (consolidation score $\geq$ 2), a high $\mathrm{FIO}_{2}$ led to higher consolidation and $\mathrm{B}$-line scores. The study compared $\mathrm{FIO}_{2}$ of 0.3 and 0.6 , which is a relatively small difference, and did not include laparoscopic surgeries in the Trendelenburg position, which may explain its discrepancy with our results. Recently, 
Table 2 Intraoperative and postoperative modified lung ultrasound scores

\begin{tabular}{|c|c|c|c|}
\hline & Low-FIO 2 group $(n=44)$ & High- $\mathrm{FlO}_{2}$ group $(n=46)$ & $P$-value \\
\hline \multicolumn{4}{|l|}{ Baseline, after intubation } \\
\hline Substantial atelectasis, $\mathrm{n}$ & $12(27.3 \%)$ & $15(32.6 \%)$ & 0.747 \\
\hline Total modified LUSS & $5.0(3.0-8.0)$ & $4.0(4.0-6.0)$ & 0.524 \\
\hline Anterior regions & $0.0(0.0-1.0)$ & $0.0(0.0-1.0)$ & 0.538 \\
\hline Lateral regions & $1.0(0.0-2.5)$ & $1.0(0.0-2.0)$ & 0.427 \\
\hline Posterior regions & $4.0(2.0-4.0)$ & $3.0(3.0-4.0)$ & 0.839 \\
\hline \multicolumn{4}{|c|}{ End of surgery, before extubation } \\
\hline Substantial atelectasis, $\mathrm{n}$ & $21(47.7 \%)$ & $39(84.8 \%)$ & $<0.001$ \\
\hline Total modified LUSS & $6.0(4.5-9.0)$ & $12.0(9.0-14.0)$ & $<0.001$ \\
\hline Anterior regions & $0.0(0.0-2.0)$ & $2.0(1.0-4.0)$ & $<0.001$ \\
\hline Lateral regions & $1.5(0.0-2.5)$ & $3.5(2.0-4.0)$ & $<0.001$ \\
\hline Posterior regions & $4.0(3.0-6.0)$ & $6.0(5.0-8.0)$ & $<0.001$ \\
\hline \multicolumn{4}{|c|}{ Post-anesthesia care unit, before discharge } \\
\hline Substantial atelectasis, $\mathrm{n}$ & $22(50.0 \%)$ & $40(87.0 \%)$ & $<0.001$ \\
\hline Total modified LUSS & $7.0(5.0-10.0)$ & $12.0(10.0-16.0)$ & $<0.001$ \\
\hline Anterior regions & $1.0(0.0-2.5)$ & $3.0(2.0-4.0)$ & $<0.001$ \\
\hline Lateral regions & $2.0(1.0-3.5)$ & $4.0(2.0-5.0)$ & $<0.001$ \\
\hline Posterior regions & $4.0(3.0-6.0)$ & $7.0(5.0-8.0)$ & $<0.001$ \\
\hline
\end{tabular}

Data are expressed as median (interquartile range), or number (\%). Anterior, lateral, and posterior regions of the thorax were divided by the anterior and posterior axillary lines. LUSS, lung ultrasound score

Cohen et al. showed that difference of $\mathrm{FIO}_{2}$ (0.3 vs. 0.8) throughout the surgery did not increase the risk of postoperative pulmonary complications [26], while we performed a randomized controlled trial to show that short exposure to high $\mathrm{FIO}_{2}$ (1.0) during the alveolar recruitment maneuver may affect postoperative atelectasis. According to Edmark et al., however, $\mathrm{FiO}_{2} 0.8$ was reported as being of borderline importance as a cause of absorption atelectasis, and they further reported that absorption atelectasis occurred when exposed to $\mathrm{FIO}_{2}$ 1.0 , even during short preoxygenation [23]. With respect to the diagnostic method, our study performed LUSS in the immediate postoperative period for all patients while Cohen et al. detected patients with pulmonary complications by reviewing diagnosis codes or events documented in the medical chart.
We observed no significant difference in the $\mathrm{PaO}_{2}$ to $\mathrm{FIO}_{2}$ ratio at any time-point. Recruitment of collapsed alveoli with high oxygen concentrations led to a rapid re-collapse of the inflated alveoli than benefiting oxygenation. In clinical practice, $\mathrm{FIO}_{2}$ may be increased during $\mathrm{RM}$ for rapid improvements in $\mathrm{SPO}_{2}$, in cases of desaturation during surgery. Nonetheless, we found that a high $\mathrm{FIO}_{2}$ during $\mathrm{RM}$ did not actually improve oxygenation, despite a transient, rapid increase in $\mathrm{SPO}_{2}$. A recent study of 32 patients undergoing laparoscopic cholecystectomy compared $\mathrm{PaO}_{2}$ levels after two times of intraoperative RM, with $\mathrm{FIO}_{2} 0.3$ and $\mathrm{FIO}_{2} 1.0$ [27]. Although the intraoperative $\mathrm{PaO}_{2}$ did not differ between the groups, it was significantly better in the $\mathrm{FIO}_{2} 0.3$ group on postoperative blood gas analysis. This finding differed from that in our study because of possible differences in

Table 3 Perioperative $\mathrm{PaO}_{2}$ to $\mathrm{FIO}_{2}$ ratio from arterial blood gas analysis

\begin{tabular}{llll}
\hline & Low-FlO group $(\mathrm{n}=44)$ & High-FiO group $(\mathrm{n}=46)$ & $P$-value \\
\hline $\begin{array}{l}\text { Baseline, preoperative } \\
\text { Intraoperative }\end{array}$ & $430.0(385.0-492.5)$ & $438.0(370.0-485.0)$ & \\
$\quad$ & & & \\
20 min after induction & $490.0(410.0-531.2)$ & $437.5(375.0-530.0)$ & 0.364 \\
20 min after Trendelenburg & $405.0(111.4)$ & $408.6(123.5)$ & 0.884 \\
$\quad 20$ min after supine & $471.8(117.4)$ & $490.8(142.2)$ & 0.492 \\
Post-anesthesia care unit, postoperative & $457.5(397.5-552.5)$ & $455.0(400.0-495.0)$ & 0.448 \\
\hline
\end{tabular}

Data are expressed as mean (standard deviation) or median (Interquartile range) 
Table 4 Intraoperative and postoperative variables

\begin{tabular}{|c|c|c|c|}
\hline & Low-FIO 2 group $(n=44)$ & High-FIO ${ }_{2}$ group $(n=46)$ & $P$-value \\
\hline \multicolumn{4}{|l|}{ Hemodynamic variables during anesthesia } \\
\hline Heart rate (beats min $^{-1}$ ) & $62.2(57.0-67.4)$ & $62.5(57.8-70.3)$ & 0.214 \\
\hline Mean arterial pressure (mmHg) & $88.8(9.3)$ & $87.8(7.8)$ & 0.586 \\
\hline Cardiac index $\left(\mathrm{L} \mathrm{min}^{-1} \mathrm{~m}^{-2}\right)$ & $2.5(2.2-3.3)$ & $2.5(2.1-3.0)$ & 0.457 \\
\hline Stroke volume variation (\%) & $9.5(3.9)$ & $10.8(3.6)$ & 0.103 \\
\hline Mean $\mathrm{SPO}_{2}(\%)$ & $99.9(99.5-100.0)$ & $99.8(99.1-100.0)$ & 0.154 \\
\hline Lowest $\mathrm{SPO}_{2}(\%)$ & $99.0(98.0-100.0)$ & $98.0(97.0-100.0)$ & 0.119 \\
\hline Intraoperative desaturation $\left(\mathrm{SPO}_{2}<95 \%\right), \mathrm{n}$ & $3(6.8 \%)$ & $2(4.3 \%)$ & 0.959 \\
\hline \multicolumn{4}{|l|}{ Respiratory parameters during capnoperitoneum } \\
\hline Minute ventilation $\left(L \min ^{-1}\right)$ & $6.3(0.9)$ & $6.4(1.0)$ & 0.563 \\
\hline Peak inspiratory pressure $\left(\mathrm{cmH}_{2} \mathrm{O}\right)$ & $23.8(3.6)$ & $23.3(3.1)$ & 0.485 \\
\hline Static compliance $\left(\mathrm{ml} \mathrm{cmH}_{2} \mathrm{O}^{-1}\right)$ & $29.7(7.8)$ & $30.1(7.3)$ & 0.776 \\
\hline \multicolumn{4}{|l|}{ Postoperative outcome variables } \\
\hline Fever within postoperative $24 \mathrm{~h}\left(>38.0^{\circ} \mathrm{C}\right), \mathrm{n}$ & $6(13.6 \%)$ & $10(21.7 \%)$ & 0.317 \\
\hline Atelectasis on postoperative chest $\mathrm{X}$-ray, $\mathrm{n}$ & $4(9.1 \%)$ & $3(6.5 \%)$ & 0.710 \\
\hline Length of hospital stay (day) & $3.5(2.0-5.0)$ & $5.0(2.0-6.0)$ & 0.096 \\
\hline In-hospital pulmonary complication, n & $0(0.0 \%)$ & $0(0.0 \%)$ & \\
\hline
\end{tabular}

Data are expressed as mean (standard deviation), median (interquartile range), or number (\%)

the mean operation time and the patients' position. During surgery in a sitting position, such as laparoscopic cholecystectomy, the atelectasis may be more affected by $\mathrm{FIO}_{2}$ than other factors, compared to in surgery performed in a Trendelenburg position.

In our study, the overall intraoperative desaturation incidence was markedly lower than that in the study of Monastesse et al.; this could be mainly due to repetitive RM [defined as $\mathrm{SPO}_{2}<95 \%$ vs. $\mathrm{SPO}_{2}<94 \% ; 5 / 90$ (5.6\%) vs. $4 / 29$ (13.8\%), excluding a case of endobronchial intubation] [21]. In our study, the $\mathrm{SPO}_{2}$ did not decrease below $90 \%$ in either group, and no patient required a rescue by a change in the $\mathrm{FIO}_{2}$ or PEEP. Furthermore, in-hospital pulmonary complications were absent in both groups. This may have been due to the inclusion of only patients with a low risk of pulmonary complications, along with repeated RM during mechanical ventilation. Postoperative fever $\left(>38^{\circ} \mathrm{C}\right)$ developed in a considerable number of patients in both groups. The length of hospital stay was non-significantly longer in the high- $\mathrm{FIO}_{2}$ group.

The postoperative modified LUSS in this study was similar to that in the study by Monastesse et al. [21]. In our study, the PACU score in the low- $-\mathrm{FIO}_{2}$ group was lower and that of the high- $\mathrm{FIO}_{2}$ group was higher than in the previous study, although the mean values in both studies were similar. We also analyzed the incidence of substantial atelectasis, which was observed in $>80 \%$ of patients in the high- $\mathrm{FIO}_{2}$ group. A higher score and consolidation were mainly observed in the posterior (dependent) part of the thorax, which can be attributed to pneumoperitoneum and the Trendelenburg position. As all patients showed at least a single, small, subpleural consolidation after pneumoperitoneum in the study of Monastesse et al. [21], this incidence of substantial atelectasis is likely to be acceptable. Nonetheless, the substantial atelectasis observed in our study did not alter the clinical outcome.

Our study had several limitations. First, ultrasound is an operator-dependent imaging modality [28], and observed findings may vary based on the operator's experience. However, the sonographers in our study were well-experienced in lung ultrasound examination, and therefore, operator-related variations were minimal. Second, since only patients with a low risk of pulmonary complications were included; therefore, our results cannot be extended to patients with lung disease. Moreover, clinical consequences of the atelectasis may not have been observed for the same reason. Third, the anesthesiologist who performed the RM was not blinded. However, the anesthesiologist performing lung ultrasound for outcome measurement was blinded to the $\mathrm{FIO}_{2}$ used for the RM. Fourth, there is a possibility of incomplete intraoperative recruitment with the opening pressure obtained in the supine state before surgical incision. The opening pressure was used as access to the dependent part of the thorax was limited during the surgery. Nevertheless, it was considered to be sufficiently 
effective because RM was mostly performed at a high pressure of $\geq 30 \mathrm{cmH}_{2} \mathrm{O}$. Fifth, we applied uniform PEEP of $5 \mathrm{cmH}_{2} \mathrm{O}$ to all patients, not an individualized PEEP. After open up the lung with RM, sufficient level of PEEP is required to keep the lung free of collapse. However, identifying the optimal PEEP is another topic that should be further discussed. Lastly, the definition of substantial atelectasis was not validated by previous studies. Although previous studies have used lung ultrasound as a diagnostic tool for atelectasis $[15,19,21,29-31]$, the criteria for substantial atelectasis are yet to be established.

In conclusion, for patients undergoing laparoscopic surgery in the Trendelenburg position, a higher LUSS, reflecting a higher degree of absorption atelectasis, was observed when RM was performed with a high $\mathrm{FIO}_{2}$ (1.0) than with a low $\mathrm{FiO}_{2}$ (0.4). We also found that using a high $\mathrm{FiO}_{2}$ during $\mathrm{RM}$ yields no oxygenation benefit and may result in more atelectasis than when using low $\mathrm{FIO}_{2}$.

\section{Abbreviations}

RM: Recruitment maneuver; PEEP: Positive-end expiratory pressure; LUSS: Lung ultrasound score; PACU: Post-anesthesia care unit

\section{Acknowledgements}

None.

\section{Authors' contributions}

Study design: BRK, J-HB, SY. Study conduct and data collection: BRK, SL, HB, ML. Data analysis: ML, SL, SY. Writing and revising paper: BRK, SL, $H B, M L, J-H B, S Y$. Final approval of the paper: All authors.

\section{Funding}

None declared.

\section{Availability of data and materials}

The datasets used and/or analyzed during the current study are available from the corresponding author on reasonable request.

\section{Ethics approval and consent to participate}

This trial was approved by the Institutional Review Board of Seoul National University Hospital (No. 1903-137-1020, 22 April 2019) and written informed consent was obtained from all participants.

\section{Consent for publication}

Not applicable.

\section{Competing interests}

The authors declare that they have no competing interests.

\section{Received: 3 May 2020 Accepted: 9 July 2020}

Published online: 18 July 2020

\section{References}

1. Gunnarsson L, Tokics L, Gustavsson H, Hedenstierna G. Influence of age on atelectasis formation and gas exchange impairment during general anaesthesia. Br J Anaesth. 1991;66(4):423-32

2. Hedenstierna G, Edmark L. Mechanisms of atelectasis in the perioperative period. Best Pract Res Clin Anaesthesiol. 2010;24(2):157-69.

3. Strang CM, Freden F, Maripuu E, Ebmeyer U, Hachenberg T, Hedenstierna G. Improved ventilation-perfusion matching with increasing abdominal pressure during CO (2) -pneumoperitoneum in pigs. Acta Anaesthesiol. Scand. 2011;55(7):887-96.
4. Andersson LE, Baath M, Thorne A, Aspelin P, Odeberg-Wernerman S. Effect of carbon dioxide pneumoperitoneum on development of atelectasis during anesthesia, examined by spiral computed tomography. Anesthesiology. 2005:102(2):293-9.

5. Duggan M, Kavanagh BP. Pulmonary atelectasis: a pathogenic perioperative entity. Anesthesiology. 2005;102(4):838-54

6. Regli A, Habre W, Saudan S, Mamie C, Erb TO, von Ungern-Sternberg BS Impact of Trendelenburg positioning on functional residual capacity and ventilation homogeneity in anaesthetised children. Anaesthesia. 2007;62(5): 451-5.

7. Brooks-Brunn JA. Postoperative atelectasis and pneumonia. Heart \& Lung: The Journal of Cardiopulmonary and Acute Care. 1995;24(2):94-115.

8. van Kaam AH, Lachmann RA, Herting E, De Jaegere A, van Iwaarden F, Noorduyn $L A$, et al. Reducing atelectasis attenuates bacterial growth and translocation in experimental pneumonia. Am J Respir Crit Care Med. 2004; 169(9):1046-53.

9. Rothen HU, Sporre B, Engberg G, Wegenius G, Hedenstierna G. Reexpansion of atelectasis during general anaesthesia: a computed tomography study. Br J Anaesth. 1993;71(6):788-95.

10. Tusman G, Bohm SH. Vazquez de Anda GF, do campo JL, Lachmann B. 'Alveolar recruitment strategy' improves arterial oxygenation during general anaesthesia. Br J Anaesth. 1999;82(1):8-13.

11. Futier E, Constantin JM, Pelosi P, Chanques G, Kwiatkoskwi F, Jaber $\mathrm{S}$, et al. Intraoperative recruitment maneuver reverses detrimental pneumoperitoneum-induced respiratory effects in healthy weight and obese patients undergoing laparoscopy. Anesthesiology. 2010;113(6): 1310-9.

12. Park HP, Hwang JW, Kim YB, Jeon YT, Park SH, Yun MJ, et al. Effect of preemptive alveolar recruitment strategy before pneumoperitoneum on arterial oxygenation during laparoscopic hysterectomy. Anaesth Intensive Care. 2009;37(4):593-7.

13. Park SH, Jeon YT, Hwang JW, Do SH, Kim JH, Park HP. A preemptive alveolar recruitment strategy before one-lung ventilation improves arterial oxygenation in patients undergoing thoracic surgery: a prospective randomised study. Eur J Anaesthesiol. 2011;28(4):298-302.

14. Song IK, Jang YE, Lee JH, Kim EH, Yoo S, Kim HS, et al. Effect of different fraction of inspired oxygen on development of atelectasis in mechanically ventilated children: a randomized controlled trial. Paediatr Anaesth. 2019; 29(10):1033-9.

15. Acosta CM, Maidana GA, Jacovitti D, Belaunzaran A, Cereceda S, Rae E, et al. Accuracy of transthoracic lung ultrasound for diagnosing anesthesiainduced atelectasis in children. Anesthesiology. 2014;120(6):1370-9.

16. Goffi A, Kruisselbrink R, Volpicelli G. The sound of air: point-of-care lung ultrasound in perioperative medicine. Can J Anaesth. 2018;65(4):399-416.

17. Touw HR, Parlevliet KL, Beerepoot M, Schober P, Vonk A, Twisk JW, et al. Lung ultrasound compared with chest $X$-ray in diagnosing postoperative pulmonary complications following cardiothoracic surgery: a prospective observational study. Anaesthesia. 2018;73(8):946-54.

18. Song IK, Kim EH, Lee JH, Kang P, Kim HS, Kim JT. Utility of perioperative lung ultrasound in pediatric cardiac surgery: a randomized controlled trial. Anesthesiology. 2018:128(4):718-27.

19. Yu X, Zhai Z, Zhao Y, Zhu Z, Tong J, Yan J, et al. Performance of lung ultrasound in detecting Peri-operative atelectasis after general anesthesia. Ultrasound Med Biol. 2016;42(12):2775-84.

20. Aldrete JA. The post-anesthesia recovery score revisited. J Clin Anesth. 1995; 7(1):89-91.

21. Monastesse A, Girard F, Massicotte N, Chartrand-Lefebvre C, Girard M. Lung ultrasonography for the assessment of perioperative atelectasis: a pilot feasibility study. Anesth Analg. 2017;124(2):494-504.

22. Abbott TEF, Fowler AJ, Pelosi P. Gama de Abreu M, Møller AM, Canet J, et al. a systematic review and consensus definitions for standardised endpoints in perioperative medicine: pulmonary complications. $\mathrm{Br} J$ Anaesth. 2018;120(5):1066-79

23. Edmark L, Kostova-Aherdan K, Enlund M, Hedenstierna G. Optimal oxygen concentration during induction of general anesthesia. Anesthesiology. 2003; 98(1):28-33.

24. Reber A, Engberg G, Wegenius G, Hedenstierna G. Lung aeration. The effect of pre-oxygenation and hyperoxygenation during total intravenous anaesthesia. Anaesthesia. 1996;51(8):733-7.

25. Rothen HU, Sporre B, Engberg G, Wegenius G, Hogman M, Hedenstierna G. Influence of gas composition on recurrence of atelectasis after a 
reexpansion maneuver during general anesthesia. Anesthesiology. 1995; 82(4):832-42.

26. Cohen B, Ruetzler K, Kurz A, Leung S, Rivas E. Ezell J, et al. Intra-operative high inspired oxygen fraction does not increase the risk of postoperative respiratory complications: Alternating intervention clinical trial. 2019;36(5): $320-6$.

27. Topuz U, Salihoglu Z, Gokay BV, Umutoglu T, Bakan M, Idin K. The effects of different oxygen concentrations on recruitment maneuver during general anesthesia for laparoscopic surgery. Surg Laparosc Endosc Percutan Tech. 2014;24(5):410-3.

28. Brandli L. Benefits of protocol-driven ultrasound exams. Radiol Manage. 2007;29(4):56-9.

29. Ferrando C, Romero C, Tusman G, Suarez-Sipmann F, Canet J, Dosda R, et al. The accuracy of postoperative, non-invasive air-test to diagnose atelectasis in healthy patients after surgery: a prospective, diagnostic pilot study. BMJ Open. 2017;7(5):e015560.

30. Girard M, Genereux V, Monastesse A. Lung ultrasonography for the detection of anesthesia-induced lung atelectasis. Anesthesiology. 2015; 122(1):213-4.

31. Lee JH, Choi S, Ji SH, Jang YE, Kim EH, Kim HS, et al. Effect of an ultrasoundguided lung recruitment manoeuvre on postoperative atelectasis in children: a randomised controlled trial. Eur J Anaesthesiol. 2020

\section{Publisher's Note}

Springer Nature remains neutral with regard to jurisdictional claims in published maps and institutional affiliations.

- fast, convenient online submission

- thorough peer review by experienced researchers in your field

- rapid publication on acceptance

- support for research data, including large and complex data types

- gold Open Access which fosters wider collaboration and increased citations

- maximum visibility for your research: over $100 \mathrm{M}$ website views per year

At $\mathrm{BMC}$, research is always in progress.

Learn more biomedcentral.com/submissions 